\title{
ACESSO Ȧ INSULINOTERAPIA DE USUÁRIOS COM DIAGNÓSTICO DE DIABETES MELLITUS ACOMPANHADOS EM AMBULATÓRIO ESPECIALIZADO
}

\author{
Mariana da Conceição Santana Lima \\ Paulo Henrique Fernandes Santos ${ }^{2}$ \\ Keila Cristianne Trindade da Cruz ${ }^{2}$ \\ Lucas Cardoso dos Santos ${ }^{3}$ \\ Valéria Bertonha Machado ${ }^{2}$ \\ Juliane Andrade ${ }^{2}$
}

\author{
https://orcid.org/0000-0002-3561-1042 \\ https://orcid.org/0000-0003-4533-0129 \\ https://orcid.org/0000-0001-8146-8323 \\ https://orcid.org/0000-0002-7337-2759 \\ https://orcid.org/0000-0003-1106-4571 \\ https://orcid.org/0000-0002-4321-0118
}

Objetivo: Caracterizar o perfil e o acesso à insulinoterapia dos usuários com diagnóstico de Diabetes Melittus atendidos no Ambulatório de Endocrinologia de um hospital universitário do Distrito Federal. Método: Estudo transversal, realizado de abril a maio de 2019. A amostra constituiu-se de 60 participantes com 18 anos ou mais. Foi aplicado questionário estruturado e os dados descritivos analisados pelo software PSPP. Resultados: Verificou-se que a maioria (86,7\%) são mulheres, que os participantes obtêm insulina (93,3\%), antidiabéticos orais (50,0\%) e insumos (93,3\%) no serviço público, e que metade desses participantes os adquirem na unidade de saúde. Ainda metade (53,3\%) relatou ter interrompido o tratamento devido à falta desses medicamentos. Conclusão: $O$ estudo evidenciou as fragilidades no acesso às redes de saúde do Distrito Federal em decorrência da falta de investimentos na Atenção Básica e da cultura hegemônica, sendo urgente o investimento nas redes de cuidado à luz das políticas de acesso.

Descritores: Acesso aos Serviços de Saúde; Diabetes mellitus; Níveis de Atenção à Saúde, Assistência Integral à Saúde

\section{ACCESS TO INSULINTHERAPY OF USERS WITH DIABETES MELLITUS ACCOMPANIED IN A SPECIALIZED OUTPATIENT CLINIC}

Objective: To characterize the profile and access to insulin therapy of users with diabetes mellitus treated in the Endocrinology Outpatient Clinic in an university hospital of Distrito Federal. Method: Cross-sectional study, performed from April to May 2019. The sample consisted of 60 participants with 18 years or more. A structured questionnaire was applied and the descriptive data analyzed by the PSPP software. Results: It was found that the majority (86.7\%) were women, that participants obtain insulin (93.3\%), oral antidiabetics (50.0\%) and inputs (93.3\%) in the public service, and that half of these participants acquire it at the health unit. Half (53.3\%) reported having interrupted the treatment due to the lack of these medications. Conclusion: The study showed the weaknesses in access to networks of health of Distrito Federal due to the lack of investment in primary health care and the hegemonic culture, being urgent the investment in care networks of care in the light of the access policies.

Descriptors: Health Services Accessibility; Diabetes Mellitus; Health Care Levels; Comprehensive Health Care

\section{ACCESO A INSULINOTERAPIA DE USUARIOS CON DIAGNÓSTICO DE DIABETES MELLITUS ACOMPAÑADO EN AMBULATORIO ESPECIALIZADO}

Objetivo: caracterizar el perfil y el acceso a la terapia con insulina de los usuarios diagnosticados con Diabetes Melittus atendidos en la Clínica Ambulatoria de Endocrinología de un hospital universitario en el Distrito Federal. Método: estudio transversal, realizado de abril a mayo de 2019. La muestra consistió en 60 participantes de 18 años o más. Se aplicó un cuestionario estructurado y los datos descriptivos fueron analizados por el software PSPP. Resultados: se encontró que la mayoría (86.7\%) son mujeres, los participantes reciben insulina (93.3\%), antidiabéticos orales (50.0\%) e insumos (93.3\%) en el servicio público, y la mitad de estos participantes los adquieren en el centro de salud. Todavía la mitad (53.3\%) informó suspender el tratamiento debido a la falta de estos medicamentos. Conclusión: El estudio mostró las debilidades en el acceso a las redes de salud en el Distrito Federal debido a la falta de inversiones en atención primaria y cultura hegemónica, siendo urgente invertir en redes de atención a la luz de las políticas de acceso.

Descriptors: Accesibilidad a los Servicios de Salud; Diabetes Mellitus; Niveles de Atención de Salud; Atención Integral de Salud

${ }^{1}$ Fundação Osvaldo Cruz- Fiocruz, Rio de Janeiro, RJ.

${ }^{2}$ Universidade de Brasilia, DF.

${ }^{3}$ Universidade Estadual Paulista/Botucatu, SP

Autor correspondente: Juliane Andrade E-mail: juenf_andrade@yahoo.com.br

Recebido: 01/11/2019 Aceito: 18/03/2020 


\section{INTRODUÇÃO}

A Organização Mundial de Saúde define Diabetes Mellitus (DM) como uma desordem metabólica de múltipla etiologia(1). Atualmente a DM é classificada, de acordo com a sua etiologia, em quatro classes clínicas: DM tipo 1, DM tipo 2, outros tipos especificos de DM e DM gestacional(2).

Quanto ao tratamento, para a DM tipo 1, necessariamente a insulina é indicada, para a DM tipo 2, a terapêutica medicamentosa (antidiabéticos orais e insulinas) é utilizada quando não há controle glicêmico por meio da alimentação nem prática regular de exercício físico ${ }^{(2)}$. O objetivo principal do tratamento da DM consiste na obtenção de qualidade de vida e longevidade ${ }^{(3)}$.

Nesse contexto é necessária uma abordagem integral ao portador de DM e sua família, que seja apoiada na Rede de Atenção à Saúde (RAS), organizada de forma integrada e contribuidora para o acesso e acessibilidade dos usuários aos serviços e à continuidade do cuidado(4).

\section{Nessa perspectiva, faz-se necessário trazer a definição de acesso e acessibilidade:}

Acessibilidade refere-se a características da oferta, sendo o aspecto que permite que as pessoas cheguem ao serviço e assim se estabeleça a atenção ao primeiro contato, e acesso a forma como as pessoas percebem a acessibilidade, ou seja como os usuários experimentam essa característica no seu serviço de saúde ${ }^{(5: 225)}$.

Desse modo, a falta de acesso no tocante à carência de medicamentos e insumos para DM pode impactar diretamente no tratamento do indivíduo. Sabe-se que a dificuldade de acesso aos medicamentos está relacionado com um pior estado de saúde, busca por outras terapias, retornos ao serviço de saúde e maior gasto com o tratamento(6).

Ao realizar atenção integral à saúde de usuários portadores de doenças crônicas, a assistência é direcionada e apoiada pelas RAS, que têm como estratégia a superação ao modo fragmentado de operar, buscando integração entre os pontos de atenção, permitindo que o usuário seja atendido em todas as suas necessidades. No entanto, quando o funcionamento desse sistema esbarra em uma cultura burocrática, desarticulada, torna-se lento e insatisfatório, impactando no cuidado ${ }^{(7)}$.

Diante do exposto, é fundamental um melhor entendimento sobre o acesso dos usuários aos medicamentos $\mathrm{e}$ insumos no tratamento da DM na realidade do Distrito Federal (DF), visando a reorganização das redes de atenção para o cuidado qualificado. Portanto, a presente investigação tem como objetivo caracterizar o perfil e o acesso à insulinoterapia dos usuários com diagnóstico de DM atendidos no Ambulatório de Endocrinologia de um hospital universitário.

\section{MÉTODO}

\section{Tipo de Estudo}

Trata-se de um estudo descritivo e transversal.

\section{Participantes da pesquisa}

A amostra foi composta pela totalidade dos pacientes em uso de insulina com diagnóstico de DM tipo l e DM tipo 2 , atendidos no Ambulatório de Endocrinologia $(n=60)$ identificados com o auxilio do responsável pelo serviço.

Os critérios de inclusão foram: ser atendido no ambulatório supracitado, ter idade igual ou superior a 18 anos, ser residente no DF ou em municípios do estado de Goiás que ficam em seu entorno, ser portador de DM tipo l e tipo 2 em uso de insulina. Nenhum participante recusou ou desistiu de participar da pesquisa.

\section{Local do Estudo}

Realizado em um Ambulatório de Endocrinologia de um hospital universitário localizado na região centro-norte do DF. Tal serviço é referência para a Atenção Básica (AB) encaminhar usuários com DM sem êxito no controle dos níveis glicêmicos, além de acolher aqueles que chegam ao serviço sem encaminhamentos.

Quanto à RAS no DF, desde 2015 foram criadas regiões de saúde com estímulo à descentralização e regionalização. Assim, atualmente as 31 regiões administrativas do DF estão divididas em sete regiões de saúde (centro-sul, centro-norte, oeste, sudoeste, norte, leste e sul), separadas de acordo com a sua localidade e características regionais( ${ }^{(8)}$.

\section{Coleta dos dados}

A coleta de dados ocorreu de abril a maio de 2019 por meio de um questionário estruturado, construído pelos pesquisadores, baseado em levantamento bibliográfico, utilizando questões da Pesquisa Nacional sobre Acesso, Utilização e Promoção do Uso Racional de Medicamentos no Brasil PNAUM-2013(9) e o Caderno 36 do Ministério da Saúde ${ }^{(10)}$. As variáveis foram analisadas em quatro blocos: perfil sociodemográfico, perfil clínico, terapêutica insulinica e acesso a medicamentos e insumos.

As entrevistas foram realizadas nos dias de retorno, após as consultas. Os entrevistados foram convidados a participar da pesquisa na sala de espera, sendo realizadas pela pesquisadora em lugar privativo, com duração média de 15 minutos. 


\section{Procedimentos de Análise dos Dados}

Os dados foram tabulados em uma planilha do programa Microsoft Excel, validados por dupla digitação e posteriormente submetidos à análise estatística descritiva com a utilização do Programa para Análise Estatística de Dados Amostrados - PSPP.

\section{Procedimentos Éticos}

Este estudo atendeu aos principios éticos estabelecidos na Resolução no 466/2012 do Conselho Nacional de Saúde e foi aprovado pelo Comitê de Ética em Pesquisa com Seres Humanos da Faculdade de Ciências da Saúde da Universidade de Brasilia com o parecer no 3.262.426 de 12 de abril de 2019 .

Os participantes foram devidamente esclarecidos sobre os objetivos e forma de participação e, para aqueles que aceitaram participar, foi solicitada a assinatura do Termo de Consentimento Livre e Esclarecido (TCLE).

\section{RESULTADOS}

Entre os 60 participantes investigados, observou-se predominância de mulheres (86,7\%), com idade entre 40 a 59 anos (45,0\%) e com 60 anos ou mais (35,0\%), sem estar casado ou com união estável (65,0\%), com menos de oito anos de estudo concluído (56,6\%) e a maioria da cor preta $(41,7 \%)$. A faixa de concentração da renda per capita familiar foi entre $\mathrm{R} \$ 500,00$ e $\mathrm{R} \$ 998,00$ (43,3\%), e, referente ao local de residência, houve representação de todas as regiões do Distrito Federal e entorno, sendo este último aquele com maior percentual (20,0\%).

Quanto ao perfil clínico, predominaram diagnósticos há mais de 15 anos (35,0\%), diabetes tipo 2 (78,3\%), mais uma comorbidade além do diabetes $(41,7)$ e ausência de complicação relacionada à diabetes (58,3\%). Quanto ao tipo de insulina utilizada, a combinação Insulina Humana Recombinante - NPH e regular foi a mais utilizada $(60,0 \%)$, seguida do uso de antidiabético oral (53,3\%).

A Tabela 1 apresenta dados referentes à terapêutica insulínica, ressaltando-se que a maior parte dos participantes referiu ser o responsável pela aplicação de insulina (86,6\%) e realizou a monitorização mais de duas vezes ao dia $(81,6 \%)$. Quanto à educação em saúde, todos mencionaram receber orientação sobre a aplicação, sendo o profissional enfermeiro o mais citado (65,0\%). A frequência de reutilização de seringas e agulhas foi de uma a oito vezes $(93,3 \%)$.

Tabela 1 - Distribuição das variáveis relacionadas à terapêutica insulínica dos usuários do ambulatório de endocrinologia do Hospital Universitário de Brasília. Brasília, DF. Brasil, 2019

\begin{tabular}{lcc} 
Variáveis terapêuticas & $\mathbf{n}$ & $\%$ \\
\hline $\begin{array}{l}\text { Responsável pela aplicação da insulina } \\
\text { Usuário }\end{array}$ & 52 & 86,6 \\
Família & 7 & 11,7 \\
Amigos & & \\
\end{tabular}

\section{Frequência da aplicação da insulina por dia}

Uma vez

6

10,0

Duas vezes

7

Mais de duas vezes

47

78,3

\section{Monitorização da glicemia capilar por dia}

Mais de duas vezes

49

81,6

Menos de duas vezes

Não monitora

\section{Orientação para utilização da insulina}

Não

Sim

60

100

\section{Profissional responsável pela orientação}

Médico

$18 \quad 30,0$

Farmacêutico

3 5,0

Enfermeiro

\section{Reutilização de seringa e agulha}

Não reutiliza

Uma a oito vezes

56

Mais de oito

1 
No que diz respeito aos componentes de acesso, demostrados na tabela 2 , verificou-se predominância de aquisição dos medicamentos no serviço público, no que se refere à insulina (93,3\%) e antidiabéticos orais (50,0\%), sendo a Unidade Básica de Saúde (UBS) o serviço de maior procura para essa aquisição. Quanto aos insumos para monitorização da glicemia capilar, glicosimetro, fita e lanceta, os participantes também declararam adquiri-los no setor público (93,3\%). A aquisição de canetas se deu pelos serviços público e privado $(23,3 \%)$, sendo o serviço público o mais utilizado (dados não mostrados na tabela 2).

Mais da metade (53,3\%) dos participantes já interrompeu o tratamento em decorrência da falta de medicamentos e insumos. Quanto à acessibilidade, a maior parte $(66,7 \%)$ não declarou dificuldade em relação ao deslocamento para o serviço de saúde (Tabela 2 ).

Tabela 2 - Distribuição das variáveis relacionadas ao acesso a medicamentos e insumos dos usuários em uso de insulinoterapia do ambulatório de endocrinologia. Brasília, DF. Brasil, 2019

\begin{tabular}{llc} 
Variáveis de acesso & $\mathbf{n}$ & $\%$ \\
\hline Serviço de aquisição de insulina $(\mathbf{n}=\mathbf{6 0})$ & & \\
Público & 56 & 93,3 \\
Privado-compra & 4 & 6,7
\end{tabular}

Serviço público de aquisição de insulina $(n=56)$

$\begin{array}{lcc}\text { UBS } & 32 & 57,1 \\ \text { Farmácia popular } & 17 & 30,4 \\ \text { Hospital } & 7 & 12,5\end{array}$

$\begin{array}{lcc}\text { Serviço de aquisição de antidiabético }(\mathbf{n = 3 3 )} & \\ \text { Público } & 30 & 91,0 \\ \text { Privado-compra } & 3 & 9,0\end{array}$

Serviço público de aquisição de antidiabético $(n=30)$

$\begin{array}{lcc}\text { UBS } & 23 & 76,7 \\ \text { Farmácia popular } & 7 & 23,3\end{array}$

\section{Utilização de glicosímetro em casa}

$\begin{array}{lcc}\text { Não } & 4 & 6,7 \\ \text { Sim } & 56 & 93,3\end{array}$

Serviço público de aquisição de glicosímetro $(n=56)$

UBS

$28 \quad 50,0$

Farmácia popular

5

9,0

Hospital

41,0

Serviço público de aquisição de fita $(n=56)$

UBS 44

78,6

Farmácia popular 2

Hospital

10

17,8

Serviço público de aquisição lanceta $(n=56)$

UBS

40

Farmácia popular 59,0

Hospital

11

19,6

Interrupção do tratamento por falta de
insumos
e medicamentos $(\mathbf{n = 6 0 )}$
$\quad$ Não
$\begin{array}{lll}\text { Sim } & 28 & 46,7 \\ & 32 & 53,3\end{array}$

Insumos e medicamentos ausentes

5

Medicamentos

Ambos

Formas de contornar a falta de insumos

$(n=32)$

Comprou

Doação

Ficou sem

Ficou internada

6,3

Variáveis

$\%$

Dificuldade de acessibilidade à UBS

$(n=60)$

Não

40

66,7

Sim

20

33,3

Motivo da dificuldade de acessibilidade ao serviço de saúde $(n=20)$

Distância 11 55,0

Distância e dependência de terceiros

420,0

Distância e gastos 


\section{DISCUSSÃO}

A presente pesquisa permitiu caracterizar o perfil e o acesso à insulinoterapia dos usuários diabéticos. Desse modo, as variáveis foram organizadas de forma a refletir os determinantes para utilização dos serviços de saúde, por meio do entendimento sobre acesso a insulina e insumos relacionados.

No que diz respeito à variável sexo, a maioria dos participantes da pesquisa era do sexo feminino. No contexto dessa pesquisa, deve-se considerar a ênfase dada por políticas públicas na atenção à saúde materno-infantil que reforçam ainda mais essa diferenciação entre os gêneros na busca pelos serviços de saúde ${ }^{(11)}$.

De modo geral, a amostra é composta por adultos, porém ressalta-se que $35 \%$ correspondem a uma população idosa. Esse achado pode ser justificado por alterações fisiológicas inerentes ao processo de envelhecimento, as quais estão relacionadas a um maior risco de morte prematura, maior associação com comorbidades e diminuição da qualidade de vida ${ }^{(2)}$

O grau de instrução foi diversificado, predominando menos de oito anos de estudo, com renda de meio salário mínimo a um salário mínimo, e declarantes da cor preta. Uma investigação sobre acesso de mulheres aos serviços preventivos na Bahia, segundo raça/cor, mostrou que desigualdades sociodemográficas e raciais estão intimamente ligadas a uma barreira de acesso ${ }^{(12)}$. Outro estudo demonstrou que qualquer barreira ao acesso terá impacto maior sobre populações socioeconomicamente desfavorecidas, aumentando, assim, a discrepância do estado de saúde entre subgrupos da população(5).

Quanto ao panorama clínico dos entrevistados, revelou-se que os usuários têm diagnóstico há mais de 15 anos e a maioria referiu não ter nenhuma complicação associada, o que contradiz o perfil de usuários atendidos em ambulatórios especializados, levando a inferir que os participantes não têm conhecimento suficiente do próprio estado de saúde ou deveriam ser atendidos na UBS.

Quanto às práticas insulínicas, os próprios entrevistados realizavam as aplicações, ideal para o indivíduo desempenhar o autocuidado. As orientações quanto ao manejo da terapia tendem a ser realizadas pelo enfermeiro, profissional mais citado pelos entrevistados. A orientação é uma ferramenta que permite promover o cuidado por intermédio da educação em saúde, contribuindo para a prevenção de agravos ${ }^{(13)}$.

Referente à utilização das seringas e agulhas, foi constatado que os participantes as reutilizavam de uma a oito vezes. Tal prática vai ao encontro da recomendação do MS no Caderno $36^{(10)}$, o qual afirma que a reutilização pode ocorrer até oito vezes, desde que pela mesma pessoa. Por sua vez, sabe-se que a SBD(2) classifica os itens como de uso único, o que faz refletir se os serviços conseguiriam suprir a demanda dos usuários diante dessa recomendação.

Para falar de acesso, é importante entender a organização da rede de cuidado ao paciente com doença crônica não transmissivel (DCNT). Essa é formada por relações horizontais, devendo ser ordenada e coordenada pela $A B$, sendo este principal centro de comunicação(14). Logo, o usuário deverá ser conduzido na rede de acordo com suas necessidades, considerando os agravos de maior relevância ${ }^{(17)}$.

No DF, como já citado, culturalmente a $A B$ não teve investimento necessário e tanto os profissionais quanto os serviços de saúde ainda estão arraigados no modelo hegemônico, não havendo uma porta de entrada bem definida ${ }^{(8)}$. A entrada nos serviços de saúde está diretamente relacionada à disponibilidade de medicamentos, insumos e localização geográfica e leva em consideração aspectos qualitativos, nos quais o acolhimento, serviços e insumos prestados e valores no território são considerados ${ }^{(16)}$.

Ainda no âmbito do acesso, na presente investigação, os entrevistados relataram adquirir os medicamentos e insumos para prática de insulinoterapia pelo Sistema Único de Saúde (SUS), sendo a maioria na UBS. Contudo, alguns adquiriram cada item em um serviço diferente, posto que as porcentagens de aquisição de insulina, antidiabéticos, glicosimetro, fita e lanceta diferiram. Tais dados encontram-se na contramão do que é previsto na Política Nacional de Atenção Básica sobre o papel da $A B^{(17)}$.

O local de pesquisa integra a rede de atenção de DCNT, no entanto foi constatado que $20 \%$ da demanda do ambulatório pesquisado é de usuários do entorno, demonstrando mais uma vez a falta de organização da rede de cuidado. Os serviços prestados pelo poder público nas regiões do entorno são precários ou mesmo insuficientes, o que, consequentemente, impõe ao DF o acolhimento dessa demanda não atendida, extrapolando a área de abrangência sob sua responsabilidade sanitária ${ }^{(18)}$

$\mathrm{Na}$ presente pesquisa, foi evidenciado que os entrevistados tiveram que interromper o tratamento pela falta de insumos e medicamentos, entretanto contornaram a situação por meio de compra com custeio próprio. O custo para usuários que adquirem apenas a insulina em farmácias comerciais é de $\mathrm{R} \$ 500,00$ a $\mathrm{R} \$ 800,00$ mensais $^{(2)}$. O paciente que retira uma parte do seu orçamento familiar para poder tratar a própria doença tem grandes impactos nos aspectos pessoais, sociais e financeiros ${ }^{(19)}$.

Outra questão é a acessibilidade trazida por Starfield(5), 
a qual possibilita que os cidadãos cheguem aos serviços, ou seja, é o elemento estrutural necessário para ter acesso. Portanto, o local de atendimento deve ser acessivel e disponivel, características não evidenciadas nesta pesquisa, visto que os entrevistados se deslocam de várias regiões administrativas para buscar acesso.

\section{Limitações do Estudo}

As limitações do presente estudo encontram-se no tamanho amostral e na utilização das respostas autorrelatadas.

\section{Contribuições para a Prática}

Tendo em vista que a atuação dos profissionais de enfermagem é imprescindivel para o fortalecimento do SUS, entende-se que os achados deste estudo fornecem a esses profissionais subsidios tanto para identificar fragilidades na consolidação das redes de atenção à saúde aos usuários com DCNT, quanto para o planejar ações destinadas a esse público específico. Além disso, promove reflexões para desenvolvimento de outras pesquisas na área de saúde e enfermagem relacionadas ao acesso à insulinoterapia dos usuários com DM.

\section{CONCLUSÃO}

A presente investigação pôde observar que, mesmo os usuários sendo acompanhados no serviço de saúde público, nem todos adquirem os insumos e medicamentos para DM na rede pública e aqueles que o fazem não conseguem acesso em um único serviço. Portanto, a $A B$ pode melhorar seu desempenho de ordenadora e coordenadora do cuidado, seguindo as diretrizes da $A B$ de resolutividade da rede de atenção à saúde no DF.

Conclui-se que acessar medicamentos e insumos é um fator fundamental para o usuário na rede de cuidados, sendo mediado por determinantes que influenciam nesse acesso, tais como as desigualdades instituidas e a cultura de saúde, que implicam muitas barreiras. Assim, é urgente o empenho do poder público na organização da rede de cuidados à saúde dos usuários à luz das políticas de acesso.

\section{CONTRIBUIÇÕES DOS AUTORES:}

Autora 1 - concepção e/ou desenho; análise e interpretação dos dados; redação do artigo; Autor 2- redação do artigo; revisão crítica; revisão final; Autora 3- redação do artigo; revisão crítica; revisão final; Autor 4 - redação do artigo; revisão crítica; revisão final; Autora $\mathbf{5}$ - concepção e/ou desenho; análise e interpretação dos dados; redação do artigo; Autora 6 - concepção e/ou desenho; análise e interpretação dos dados; redação do artigo; revisão crítica; revisão final.

\section{REFERÊNCIAS}

1. World Health Organization (WHO). Definition, diagnosis and classification of diabetes mellitus and its complications. Part 1: Diagnosis and classification of diabetes mellitus. [Internet]. 1999 [acesso em 06 May 2019]. Disponivel em: https://doi. org/10.1002/(SICI)1096-9136(199807)15:7<539::AID-DIA668>3.0.CO;2-S

2. Sociedade Brasileira de Diabetes (SBD). Diretrizes da Sociedade Brasileira de Diabetes. [Internet]. 2017 [acesso em 27 set 2018]. Disponivel em: https://www.diabetes.org.br/profissionais/images-/2017/diretrizes/diretrizessbd20172018.pdf.

3. Salci MA, Meireles BHS, Silva DMGV. Educação em saúde para prevenção das complicações crônicas do diabetes mellitus na atenção primária. Esc Anna Nery. [Internet]. 2018 [acesso em 27 set 2018]; 22(1). Disponivel em: http://dx.doi.org/10.1590/21779465-EAN-2017-0262.

4. Colimoide FP, Meira MDD, Abdala GA, Oliveira SLSS. Integralidade na perspectiva de enfermeiros da Estratégia Saúde da Familia. Rev. bioét. [Internet]. 2017 [acesso em 27 set 2018]; 25(3). Disponível: http://dx.doi.org/10.1590/1983-80422017253218.

5. Starfield B. Atenção Primária: equilibrio entre necessidades de saúde, serviços e tecnologia. [Internet] Brasília: UNESCO 2002 [acesso em 27 set 2018]. Disponivel em: http://bvsms.saude.gov.br/-bvs/publicacoes/atencao_primaria_pl.pdf.

6. Boing AC, Bertoldi AD, Boing AF, Bastos JL, Peres KG. Acesso a medicamentos no setor público: análise de usuários do Sistema Único de Saúde no Brasil. Cad. Saúde Pública. [Internet]. 2013 [acesso em 06 jun 2019]; 29(4). Disponivel: http://dx.doi. org/10.1590/SO102-311X2013000400007 
7. Peiter CC, Santos JLG, Lanzoni GMM, Mello ALSF, Costa MFBNA, Andrade SR. Healthcare networks: trends of knowledge development in Brazil. Esc Anna Nery. [Internet]. 2019 [ acesso em 27 Aug 2019]; 23(1) Disponivel em: http://dx.doi. org/10.1590/2177-9465-ean-2018-0214

8. Fonseca HLP. A Reforma da Saúde de Brasilia. Ciênc. Saúde Coletiva. [Internet]. 2019 [acesso em 06 jun 2019]; 24(6). Disponivel em: http://dx.doi.org/10.1590/1413-81232018246.07902019.

9. Ministério da Saúde (BR). Secretaria de Ciência, Tecnologia e Insumos Estratégicos. Componente populacional: introdução, método e instrumentos/Série Pnaum - Pesquisa Nacional sobre Acesso, Utilização e Promoção do Uso Racional de Medicamentos no Brasil (Caderno 1). Departamento de Ciência e Tecnologia. Brasília: Ministério da Saúde; 2016 [acesso em 04 jan 2018]. Disponivel em: http://bvsms.saude.gov.br/bvs/publicacoes/componente_populacional_introducao_metodo_ instrumentos.pdf

10. Ministério da Saúde (BR). Secretaria de Atenção à Saúde. Departamento de Atenção Básica. Estratégias para o cuidado da pessoa com doença crônica: diabetes mellitus. (Cadernos de Atenção Básica, n. 36). Departamento de Atenção Básica. Brasilia: Ministério da Saúde; 2013 [acesso em 04 jan 2018]. Disponível em: https://bvsms.saude.gov.br/bvs/publicacoes/ estrategias_cuidado_pessoa_diabetes_mellitus_cab36.pdf

11. Assis MMA, Jesus WLA. Acesso aos serviços de saúde: abordagens, conceitos, políticas e modelo de análise. Ciênc. saúde coletiva. [Internet]. 2012 [acesso em 06 jun 2019]; 17(11). Disponivel em: http://dx.doi.org/10.1590/S1413-81232012001100002.

12. Goes EF, Nascimento ER. Mulheres negras e brancas e os níveis de acesso aos serviços preventivos de saúde: uma análise sobre as desigualdades. Saúde debate. [Internet]. 2013 [acesso em 06 jun 2019]; 37(99). Disponivel em: http://dx.doi. org/10.1590/S0103-11042013000400004.

13. Oliveira PS, Bezerra EP, Andrade LL, Gomes PLF, Soares MJGO, Costa MML. Atuação dos enfermeiros da estratégia saúde da familia na prevenção do pé diabético. Care Online. [Internet]. 2016 [acesso em 06 jun 2019]; 8(3). Disponivel em: http:// dx.doi.org/10.9789/2175- 5361.2016.v8i3.4841-4849.

14. Oliveira NRC. Redes de Atenção à Saúde: a atenção à saúde organizada em redes. [Internet] São Luís: UFMA; 2016 [acesso em 27 set 2018]. Disponivel em: http://www.unasus.ufma.br/site/files/livros_isbn/isbn_redesOl.pdf.

15. Ministério da Saúde (BR). Portaria no 483, de 1o de abril de 2014. Redefine a Rede de Atenção à Saúde das Pessoas com Doenças Crônicas no âmbito do Sistema Único de Saúde (SUS) e estabelece diretrizes para a organização das suas linhas de cuidado. Diário Oficial da União, 2 abr 2014. Seção l.p 50

16. Penchansky DBA, Thomas JW. The concept of access - definition and relationship to consumer satisfaction. Med Care. [Internet]. 1981 [acesso em 06 jun 2019]; 19(2). Disponivel em: https://www.ncbi.nlm.nih.gov/pubmed/7206846.

17. Almeida PF, Medina MG, Fausto MCR, Ligia G, Bousquat A, Mendonça MHM. Coordenação do cuidado e Atenção Primária à Saúde no Sistema Único de Saúde. Saúde debate. [Internet] 2018 [acesso em 06 jun 2019]; 42(1). Disponivel em: https://doi. org/10.1590/0103-11042018S116

18. Tonhá ACM. Acesso aos serviços de saúde nos municipios do entorno sul do Distrito Federal. Rev. Eletr. Enf. [Internet]. 2015 [acesso em 06 jun 2019]; 17(2). Disponivel em:

http://dx.doi.org/10.5216/ree.v17i2.29422.

19. Castro ARV, Grossi SAA. Custo do tratamento do diabetes mellitus tipo l: dificuldades das famílias. Acta paul. enferm. [Internet] 2008 [acesso em 06 jun 2019]; 21(4). Disponivel em: http://dx.doi.org/10.1590/S0103-21002008000400014. 